\title{
EI rol de las marcas fabricantes ante las demandas sociales. Análisis del anuncio de cambio de marca de los productos "Negrita" por la empresa Alicorp
}

\section{The role of manufacturer brands in the face of social demands. Analysis of "Negrita" re-branding advertising by Alicorp}

Recibido: 05/08/2020

Aceptado: 06/11/2020

Publicado: 30/11/2020

\author{
Viviana Rivas Gonzales \\ vrivasg@pucp.edu.pe \\ https://orcid.org/0000-0001-5071-4560 \\ Pontificia Universidad Católica del Perú \\ Carolina Tello Giusti \\ carolina.tello@pucp.edu.pe \\ https://orcid.org/0000-0002-5169-1679 \\ Pontificia Universidad Católica del Perú
}

Resumen: Partiendo del análisis del pronunciamiento de la marca Alicorp sobre el cambio de nombre de su marca comercial "Negrita", se propone profundizar en las razones por las cuales las marcas han comenzado a tomar posiciones políticas a través de sus discursos y acciones, y de qué manera estas decisiones impactan en la valoración de la marca fabricante, la marca comercial y sus consumidores. Aunque este interés acerca de las representaciones sociales en la publicidad no es un tema nuevo: ¿será posible que las marcas y la publicidad hayan adoptado un rol como actores sociales?, ¿son las transformaciones sociales actuales tan fuertes que demandan que las marcas tomen una posición política frente a estas? Mediante la respuesta a estas preguntas 
este análisis busca, a través de la presentación y discusión del caso, indagar en las raíces y consecuencias de este nuevo posicionamiento de marca.

Palabras clave: Posicionamiento de marca, Propósito de marca, Cambio social, Estereotipos, Alicorp.

Abstract: Based on the analysis of Alicorp's official statement regarding the renaming of their commercial brand "Negrita", this article suggests delving into the reasons why brands have begun to take political stands through their discourse and actions, and how these decisions have an impact on the commercial and manufacturers' brand equity as well as their consumers. Albeit interest regarding role portrayals in advertising is not a new issue: iIs it possible that brands and advertising have embraced a new role as social actors? ¿Are societal changes so powerful that they call for a brand to take a political stand towards them? Through on the outcome of these questions, and through this case-study discussion, this study seeks to explore the roots and consequences of this new brand positioning.

Key words: Brand positioning, Brand purpose, Social change, Stereotypes, Alicorp.

\section{Introducción}

La evolución de la publicidad ha llevado a que actualmente se piense en las marcas como "seres humanos" de los cuales esperamos comportamientos coherentes, con principios, valores y propósitos definidos. Sin embargo, cuando las marcas ejecutan acciones que nos interpelan como sociedad, los diversos actores sociales del mundo publicitario reaccionan de maneras sumamente diversas. Reacciones que van desde la reflexión y construcción de mensajes (que aplauden el cambio) hasta la justificación, bajo el paraguas de costumbre o tradición de la cual sentirnos orgullosos.

Como consecuencia de esto, las empresas establecen estrategias de marketing y comunicación publicitaria basadas en el conocimiento del contexto actual y del público objetivo con el que interactúan. Por este motivo, los aspectos económicos, sociales y políticos pueden afectar a personas, a marcas o a la 
relación entre estas. En consecuencia, las marcas se ven permanentemente interpeladas por los mensajes que transmiten, es decir, coexisten con opiniones a favor y en contra de sus propuestas. Esto hace que estén constantemente expuestas a un meticuloso escrutinio por las personas que las consumen y conocen. Es por esto que deben decidir cuál es su posición con respecto a temas sociales que puedan impactar directamente a las personas que consumen sus productos o servicios.

En los últimos años, se han presentado grandes cambios sociales relacionados con minorías que durante mucho tiempo han visto limitados sus derechos o han gozado de menos privilegios que otros grupos sociales. Es así como mujeres, afrodescendientes, latinos, la comunidad LGBTIQ etc., toman protagonismo en la comunicación publicitaria, la cual ha sabido prestar atención a estos cambios para incorporarlos como parte del discurso de marca. Sin embargo, a pesar de estas consideraciones, surgen las siguientes interrogantes: ¿son las transformaciones sociales actuales tan fuertes que demandan que las marcas tomen una posición política frente a estas?, ¿será posible que el tomar acción o no tomarla afecte la relación entre la "empresa-marca" y la "persona-consumidor”?, ¿será posible que las marcas y la publicidad hayan adoptado un rol como actores sociales?

\section{El propósito de marca como intento de conexión con el consumidor}

Cuando una empresa se asume como un actor social importante, resulta trascendental definir el rol que quiere desempeñar. Se puede apreciar en la evolución constante de las empresas y los diversos conceptos (del marketing y la publicidad) que se han ido incorporando como parte fundamental en la creación y gestión de marcas fuertes. Uno de esos conceptos es el que conocemos como "propósito de marca", definido como la razón de ser de la "empresa-marca" comercial o del "producto-marca" con respecto a sus clientes y sociedad donde se desenvuelve.

Según Livingstone (2019), "el objetivo del propósito de la marca es cambiar el mundo para mejor (la mayor parte del tiempo). A través de un propósito, las marcas se están convirtiendo en más que una marca de calidad o una expresión abstracta de sí mismas, están tomando medidas directas". Por ende, podemos afirmar que las marcas tienen un deber que cumplir hacia sus con- 
sumidores, pero también son responsables de actuar de manera en la que sus acciones no se vean sólo reflejadas en las ventas. Estas deben estar respaldadas por el propósito que se planteen las mismas. En todo caso, esta creciente importancia del propósito de una marca no es gratuita. Los públicos a los que están expuestas han aumentado y también han cambiado. Si bien el nivel de exposición mediática, disponibilidad y alcance han sido siempre constantes, estos factores hacen que hoy sea mucho más relevante monitorear permanentemente el impacto de las marcas en estos grupos.

En la actualidad, los públicos a los que está expuesta una marca se han ampliado, abarcando consumidores, accionistas, medios, el gobierno, la sociedad civil organizada y la comunidad. Para todos estos, una empresa-marca debe asegurarse de cumplir un rol fundamental con impacto positivo. Puede ser que una persona no sea el consumidor directo de una marca, pero eso no quiere decir que no pueda ser su principal crítico, detractor convencido y feroz opositor cuando esta invade su entorno, sus convicciones o su comunidad. Los catalizadores de dichas reacciones pueden ser desde una mala experiencia con un producto hasta problemas de estereotipación y gentrificación.

Mobolade (2016) señaló como principales resultados que "más del 85\% de los millennials ven su intención de compra influenciada por los esfuerzos de responsabilidad social de una marca sobre otra ... Además, el 73\% está dispuesto a probar un nuevo producto si la marca apoya una buena causa. Por lo tanto, tener un propósito de marca sólido se traduce en un incremento en la intención de compra de este segmento". Además, si hablamos de los centennials, podemos afirmar que "comparten puntos de vista, acciones y creencias ... es importante que las marcas entreguen los mensajes a estos para tener una relación positiva con las partes interesadas, una reputación positiva y seguir siendo marcas éticas" (Uche, 2018).

Este no es un resultado atípico si recolectamos los resultados de múltiples estudios sobre el perfil de millennials y centennials, que configuran la base fuerte de la fuerza trabajadora de los diversos países del mundo, incluido el Perú. Así, son el segmento de consumo que tendrá el poder adquisitivo, así como el poder sobre las decisiones de compra. En esa línea, en los últimos años, un 13\% de las compañías han reformulado su "propósito-misión" y un 53\% lo han cambiado casi en su totalidad, según EY Beacon Institute (2017). 


\section{Nuevas relaciones en la comunicación "marca-personas"}

La evolución tecnológica y la transformación de las comunicaciones han redefinido la relación en la comunicación entre marcas y personas, siendo las redes sociales los principales actores en la transformación del rol de los medios y la verticalidad con la que se distribuye la información. Hoy hay conceptos que toman fuerza y resulta importante definirlos para establecer las nuevas relaciones de la comunicación entre marcas/empresas y personas/ clientes.

Empezamos definiendo el concepto de "horizontalidad”. Según García Avilés (2015), las redes sociales horizontales son aquellas que admiten una participación libre de quien lo desee, permitiéndole entrar e interaccionar sin una temática predefinida. Como afirma Antón (2015), una participación horizontal es aquella donde todos los usuarios tienen las mismas posibilidades de obtener visibilidad y pueden interactuar de la misma forma.

Interpretamos entonces que las redes sociales permiten la interacción con las mismas oportunidades de alcance y visibilidad entre empresa-marca y personas-consumidores. El espacio digital se vuelve la plataforma para exponer opiniones de igual a igual. En ese sentido, no es más la empresa multimillonaria, transnacional (por ejemplo) versus el ciudadano de a pie, eliminando variables que antes podrían ser consideradas un problema: la ubicación, el tiempo y la velocidad de respuesta. Por esto, Sainz de Medrano (2017) asegura que existe un nuevo modelo de comunicación social, el "modelo horizontal", dado que las tecnologías digitales han provocado este salto histórico.

Asimismo, debemos definir el concepto "inmediatez". Kelly (2012) explica que la percepción de inmediatez en las comunicaciones online reduce psicológicamente la distancia entre las personas que están conversando. La evolución de las TIC ha sido fundamental para redefinir la necesidad de reducir los tiempos de respuesta en las diversas comunicaciones. En la actualidad, parece imposible soportar la espera para cualquier tipo de comunicación: personal, laboral, comercial, etc. Cómo describe Kelly (2012), la réplica instantánea también propicia un sentimiento de comodidad e induce a pensar que la persona con la que estamos hablando (en tiempo real) es más sociable que aquella que tarda más en responder. 
Es en este punto que resulta interesante cruzar los aportes de Sibilia (2006), quien afirma lo curioso que es el uso del adjetivo "real" (utilizado como "tiempo real”) para expresar "el aquí y el ahora” del plano analógico. Es así que las comunicaciones digitales "anulan las distancias geográficas sin necesidad de desplazar el cuerpo e inauguran fenómenos típicamente contemporáneos como la 'telepresencia' o la 'presencia virtual'” (Sibilia, 2006).

Por otro lado, el concepto "creación de comunidad" adquiere importancia, dado que una característica inherente a los seres humanos es su comportamiento social. Debido a esta característica, hemos creado comunidades en los entornos físicos, pero también en entornos virtuales que carecen de límites geográficos y congregan a quienes comparten intereses en común haciendo mucho más sólido su vínculo. Vivimos en una "sociedad en red" (Castells, 1997) desde finales de los años noventa y formamos parte de diversas comunidades (tanto físicas como virtuales) que nos permiten desarrollarnos, apoyarnos y sentirnos parte de un colectivo.

Por último, definiremos el término "viralización" para graficar el impacto y difusión orgánica que puede lograr una publicación en los medios sociales. Cada vez es más importante el alcance de las publicaciones, definido por la posibilidad de multiplicar su exposición sin generar costos para las empresas anunciantes. Sobre este tema Dafonte-Gomez (2014) señala que la aplicación del concepto de viralidad a la comunicación comercial ha representado para las marcas una oportunidad para franquear las tradicionales barreras de la audiencia ante la publicidad y convertirla en transmisora activa de los mensajes de la marca, mientras que Rushkoff (1994) explica que la viralidad surgió como una herramienta del marketing que pretendía facilitar y compartir mensajes o contenidos publicitarios entre los contactos. Sin embargo, el efecto multiplicador actual es mucho mayor y lo puede lograr una marca anunciante o un consumidor si el contenido es relevante, disruptivo, motivador o incitador.

De esta manera, podemos señalar que la horizontalidad, la inmediatez en la comunicación, la construcción de comunidad y la viralización de contenidos nos permiten plantear tres aspectos fundamentales sobre los que hoy se debe construir la comunicación entre marcas y personas: 
- Inmediatez con asertividad: es importante prestar atención a la implementación de espacios de interacción entre marcas y personas soportada en la inteligencia artificial que busca minimizar los puntos de estrés que produce la demora de una respuesta oportuna. Si bien el uso de chatbots permite una respuesta en tiempo real, no siempre es lo más acertado, sobre todo si buscamos empatizar con las personas-consumidores de nuestros productos o servicios. Una posibilidad es limitar el uso de los chatbots y la programación de respuestas automatizadas sólo para casos de procedimientos secuenciales posibles de determinar mediante árboles de decisión. Por otro lado, la comunicación persona a persona (por cualquier medio) permite siempre dar respuestas más humanas, a pesar de estar predeterminada y estandarizadas por la empresa.

- Equilibrio de poderes: las personas no son más simples receptores de la comunicación, utilizan sus propias redes para generar viralización e impacto, también aplican la técnica del jiu-jitsu al utilizar la fuerza de su oponente a su favor, en este caso, la gran cantidad de seguidores y fans que tienen las marcas gracias a su inversión publicitaria. Así, una persona puede repercutir en la imagen y percepción de la marca ante la audiencia. El alcance que logra, ya no es sólo en el entorno cercano de cada individuo: hoy pueden alcanzar niveles de exposición (gracias a la viralización) iguales que los anunciantes con una gran inversión publicitaria.

- Honestidad y coherencia: si la marca está trabajando para fortalecer su imagen debe pensar con claridad cuáles son los puntos con los que puede comprometerse, de tal forma que su discurso no quede divorciado de sus acciones. Hay que tener en cuenta que cuando hablamos de las acciones nos referimos a todo tipo de comportamiento, con todos sus públicos: empleados, clientes, socios, comunidad, medios, etc. Es posible que una actuación incongruente se convierta fácilmente en contenido viralizable y el impacto sea igual o mayor, pero de forma negativa.

De esta forma planteamos los nuevos términos sobre los que se construyen las comunicaciones entre las empresas-marcas y las personas-consumidores, siendo la base para la construcción de una relación. 


\subsection{Tipos de relaciones}

El campo de las relaciones entre los consumidores y las marcas ha estado en constante desarrollo desde principios de los noventas. Blackston (1992) y Fournier (1994) fueron los primeros en establecer un nexo entre las relaciones interpersonales y las marcas. Mientras que Blackston (1992) introdujo la idea de que las marcas se comportan como si fueran agentes activos dentro de la relación, Fournier (1994) llevó este concepto más allá, al proponer que las relaciones entre el consumidor y la marca pueden ser parte integral de la estrategia de la misma.

Desde entonces, la metáfora de la relación "consumidor-marca" ha dominado el pensamiento y práctica de marketing (Fournier, 1998) y una gran cantidad de bibliografía se ha escrito sobre el tema. Desde los sentimientos de amor que un consumidor puede desarrollar hacia una marca (Bruns et al., 2014; Ahuvia, 2005; Aron \& Aron, 1996) hasta la teoría de "autoexpansión" (Bechara et al., 2012), la investigación realizada (por especialistas en marketing y psicólogos sociales) documenta a fondo la forma en la que un consumidor crea relaciones significativas con las marcas y las mantiene en el tiempo.

Cada vez que un consumidor interactúa con una marca de forma personal, por ejemplo, haciéndose amigo de una marca en las redes sociales o sentirse feliz al comprar sus productos, esta interacción demuestra que las marcas son un aspecto importante de la identidad. Según el modelo de autoexpansión, al iniciar una relación "tienden a considerar al agente cercano como parte de sí mismos" (Bechara et al., 2012). Por tanto, existe un deseo compartido de adquirir las características presentadas por la marca, características que uno identifica como deseables. Primero, se crea la relación entre un consumidor y una marca siendo ahí cuando la identidad de esta se vuelve parte de la identidad del consumidor.

Al hablar de las relaciones del consumidor y una marca, Fournier (1998) afirma que "las marcas no son objetos pasivos, las marcas son y sirven como posibles compañeros dentro de una relación". La autora afirma una teoría que legitima a la marca como compañera del consumidor, además analiza cómo este último la comienza a ver como una persona. Estas interacciones (creadas por el consumidor con el mundo no material) se explican por medio de teorías que responden por qué los humanos tienden a sentir la necesidad de antropomorfizar objetos para interactuar más fácilmente con ellos. 
Así, cuando se humaniza una marca, esta adquiere el poder para “desempeñar un papel central en la vida del consumidor, lo que potencialmente le permite proyectar un aspecto que podría darle una sensación de comodidad por haber encontrado una marca que encaja con ellos" (Ahluwalia et al., 2009). La personalidad, el tono de voz y la fidelidad son algunas características similares a las humanas que son asignadas a las marcas a la hora de definirlas y trabajar con ellas. Probablemente los consumidores de Coca-Cola definirían la marca como "feliz" y "creativa", mientras que los propietarios de Harley-Davidson podrían usar palabras como "aventurero" y "libre" para definir no sólo la marca, sino también a ellos mismos.

Otro enfoque interesante es el propuesto por Blackston (1992), quien define la relación con la marca como "una extensión lógica de la idea de una personalidad de marca". Para el autor, la diferencia entre el enfoque de una "imagen de marca unidimensional" y el enfoque de la "relación de marca" se basa en la forma en que los especialistas en marketing hablan con los consumidores: mientras que el primero pregunta a los consumidores qué piensan sobre una marca, el segundo pregunta a los consumidores qué es lo que creen que la marca piensa de ellos.

\subsection{Factores que propician el cambio en las relaciones}

Una conducta de marca categorizada como "mala" puede dañar su imagen y reputación. Asimismo, este tipo de comportamiento puede tener consecuencias importantes para la relación entre el consumidor y la marca. Las consecuencias negativas ocurren no sólo por la calidad de la relación "consumidor-marca", sino también por la relación previa y la intención de recompra. Además, el alcance de los efectos de una mala conducta depende del contexto de la relación (Aaker et al., 2004).

Mientras más larga haya sido la relación con la marca, el deseo del consumidor por mantenerla será mayor. Esto se debe a que esta relación lo beneficia en el ámbito psicológico y social. Además, la confianza en la marca aumenta la probabilidad de generar una relación duradera, lo que fortalece la relación entre ambos, ya que las relaciones más largas reducen la probabilidad de decepción. 
Sin embargo, si la marca se comporta de forma contraria a la expectativa de los consumidores es muy probable que los consumidores cambien de marca a largo plazo. Esto se dará como consecuencia al deterioro del apego emocional a su marca. Los mensajes contradictorios a la experiencia de comunicación que ha mantenido la marca con el consumidor son interiorizados como una "mala conducta" y, por ende, castigados por los consumidores, ya que ven traicionada la confianza depositada en la marca durante todo el tiempo que han mantenido una relación con la misma.

Por otro lado, es importante notar que el volver a reforzar los mensajes originales de marca debería minimizar los posibles efectos negativos causados por la mala conducta de la marca (Aaker et al., 2004). Sin embargo, Fournier (1998) señala que una conducta de marca poco congruente no necesariamente tiene el potencial de afectar una relación de marca fuerte, ya que muchas veces el consumidor no atribuye todos los componentes negativos percibidos de una mala conducta a su marca favorita. Por ende, las personas pueden reaccionar ante el cambio propuesto por las marcas de distintas formas, dependiendo del impacto directo, preferencia por la marca, contexto y relación con las mismas.

\section{Del propósito de marca a los cambios sociales}

Los cambios sociales han sido siempre un factor constante en el desarrollo de todas las disciplinas, lo cual compromete directamente a la publicidad. Las comunicaciones (al ser una especialidad que abarca un universo tan extenso) se ven afectadas en todas sus aristas por lo que es un tema que debe abordarse de una manera particularmente especial. Felder (1999), se aproximó al concepto de "cambio social" de la siguiente manera:

La comunicación para el cambio social se define como un proceso de diálogo privado y público, a través del cual las gentes deciden quiénes son, qué quieren y cómo pueden obtenerlo. Por cambio social entendemos un cambio en la vida de un grupo social, de acuerdo a los parámetros establecidos por ese mismo grupo. Esta aproximación busca especialmente mejorar las vidas de los grupos marginados (tanto política como económicamente), y está guiada por los principios de la tolerancia, autodeterminación, equidad, justicia social y participación activa de todos. (Felder, 1999) 
La sociedad no es un ente estático, está mutando constantemente. Esto se debe a diversos factores como a la manera en la que el pensamiento de las personas que la conforman, a los avances en la tecnología, entre otros. Estos cambios generan consecuencias de carácter profundo y de largo plazo. Los resultados de estos se ven reflejados hasta el día de hoy. Tal es el caso de los movimientos sociales para promover los derechos humanos, los derechos de las mujeres, los derechos de la comunidad LGTBIQ y el movimiento "Me Too", por nombrar algunos. Una frase que se usa mucho cuando estos cambios están gestándose es "sé el cambio que quieres ver en el mundo", lo que conlleva a pensar "¿qué clase de cambios son los que queremos ver?"

Como resultado, vemos que estos cambios se manifiestan de diversas maneras y crean, a su vez, subgrupos dentro del ámbito social, de cómo se interrelacionan las personas entre ellas y con el entorno que las rodean. Sin embargo, el que existan cambios sociales "globales", no quiere decir que todas las sociedades estén listas para asimilarlos. Esta asimilación dependerá del punto en la que cada una se encuentre y de la disposición de las personas para aceptar el mismo.

Si hablamos del caso peruano, actualmente estamos viviendo la "desaparición del concepto tradicional de familia" (Arellano, 2017). Esta nueva familia propone un cambio al modelo vertical y concepto general de familia que se maneja en el país, en donde el hombre es la cabeza de la familia, el proveedor, y la mujer es representada como la responsable de las tareas que se dan dentro del hogar. Según un estudio de IPSOS (2019), estamos siendo testigos de una combinación de estos parámetros.

El Perú es un país en donde el machismo, clasismo, la homofobia y el racismo son todavía constantes que no tienen una fecha de término definida. Sin embargo, la transformación social no se ve tan distante ya que podemos ver como en la actualidad se está gestando este cambio de forma mucho más clara por parte de diferentes grupos minoritarios y las demandas sociales y políticas de estos.

Solo en el caso del racismo vemos que, según el informe del Ministerio de Cultura (2018), el 53\% de los encuestados considera que los peruanos son "racistas" o "muy racistas" y uno de cada tres personas afirman haber sido discriminado en diferentes espacios y por diversos motivos. En la misma 
encuesta, el $60 \%$ percibe que la población afroperuana es "discriminada" o "muy discriminada" por su color de piel, sus rasgos faciales o físicos y porque son asociados a la delincuencia.

En consecuencia, nos preguntamos: ¿qué están haciendo las marcas en el Perú con respecto a las transformaciones sociales?, ¿cuál es el rol que tienen como actores dentro del entorno de las comunicaciones como anunciantes y productores de contenido?, ¿será que ha llegado el momento de fusionar el rol comercial con el rol político y se está dando paso a la conformación de nuevas marcas "polimerciales"?

\section{Actores sociales de la publicidad}

Para poder entender la importancia y relevancia del caso, es importante identificar, en primer lugar, los actores sociales dentro de la publicidad impactados por el caso. Para este fin, se definirán cada uno de ellos utilizados en este análisis.

- Marca fabricante: es la empresa que posee un portafolio de productos o servicios puesto a disposición de sus clientes.

- Marca comercial: es el nombre de una línea de productos, un producto o servicio que se ofrece para un determinado público objetivo.

- Agencias de publicidad: proveedor de servicios de comunicación publicitaria, responsable de la creación de conceptos creativos, naming, posicionamiento, desarrollo de campañas entre otros servicios.

- Consumidores: clientes que usan o consumen los productos o servicios de una determinada marca comercial y pueden tener distintos vínculos con ellas: fiel, habitual, esporádico, nuevo, etc.

- Clientes potenciales: personas que pertenecen al mercado meta de una marca, comparten características del público objetivo, pero aún no consumen el producto o servicio; podrían haberlo probado, pero no lo han recomprado aún.

- La comunidad: espacio determinado por las personas pertenecientes a un mismo ámbito geográfico donde se desarrolla la actividad comercial de la marca.

- Sociedad civil organizada: grupos o comunidades formadas por intereses comunes, pueden ser de diversos tipos, desde la comunidad de vecinos hasta el colectivo (ejemplo: \#niunamenos). 


\section{El caso "Negrita": el papel de la publicidad como actor de cambio social}

¿Por qué una empresa de consumo masivo decide, en plena pandemia mundial, lanzar un comunicado anunciando el futuro cambio de su marca comercial de insumos para postres, instantáneos y postres preparados? Revisemos primero, los antecedentes de la marca fabricante y los de la marca comercial.

La marca fabricante es Alicorp, empresa peruana integrante del Grupo Romero, creada en el año 1997. Es dueña de las marcas más emblemáticas de consumo masivo en el Perú. Según su reporte anual de resultados, sólo en el año 2019 logró un crecimiento del 19,1\%, con una facturación anual de S/. 9,872 millones (US\$2,898 millones) en sus ventas netas y realizó nueve lanzamientos de productos nuevos (Alicorp, 2019).

Según el reporte anual del año 2018, el negocio de consumo masivo logró crecer 8,3\% lo que significó que 15 de las 19 marcas de su portafolio crecieran. Su cartera de productos comprende más de 125 marcas propias entre las que destacan Alacena, Don Vittorio, Blanca Flor, Cereales Ángel, Bolívar, entre otras, las cuales abarcan categorías desde detergentes hasta marcas de productos instantáneos (Alicorp, 2018). Sólo en el negocio de consumo masivo, en el año 2019, en el Perú, creció 20,6\% en ventas netas con respecto al año anterior a pesar de la desaceleración de la economía nacional.

Alicorp nació en 1956 bajo el nombre de Industrias Anderson, Clayton \& Co. (ACC), empresa fabricante de aceites y jabones. En el año 1971, el Grupo Romero adquirió Anderson Clayton \& Company y cambió su nombre a Compañía Industrial Perú Pacífico S. A. (CIPPSA); y en el año 1990 CIPPSA se fusionó con la empresa Calixto Romero S. A. y Oleaginosas Pisco S. A; ambas empresas también del Grupo Romero. Hacia 1995 adquieren las empresas La Fabril S. A. y Consorcio Distribuidor S. A. y cambia de nombre a Consorcio de Alimentos Fabril Pacífico. En 1996 adquieren las empresas Nicolini y Molinera del Perú, incrementando su participación en las categorías de harinas, fideos, alimentos balanceados y cereales. En el año 1997 cambia de nombre oficialmente a Alicorp S. A. A.

Entre los años 2005 y 2013 se expandió en Latinoamérica llegando a tener presencia en Ecuador, Argentina, Chile, Colombia y Brasil; donde hasta el día 
de hoy incorporan marcas locales a su portafolio de productos, así como también internacionalizan marcas peruanas. Entre los años 2014 y 2018 han diversificado su negocio, orientados al B2C como al B2B, adquiriendo nuevas marcas, desarrollando nuevos negocios y soluciones. Como se puede apreciar, su estrategia es principalmente comprar marcas ya desarrolladas y con una participación significativa en los territorios donde tiene presencia. Es a través del uso de sus canales de distribución nacional y su economía de escala que explotan las marcas y desarrollan al máximo el potencial de las mismas.

En el ranking Merco, Alicorp ocupa el séptimo lugar (Monitor Empresarial de Reputación Corporativa, 2019) superando a otras empresas de consumo masivo como Nestlé (noveno), Kimberly-Clark (décimo) y Procter \& Gamble (décimo quinto). Según América Economía (2019), Alicorp figura en décimo segunda posición, superada sólo por empresas del rubro financiero, retail o actividades extractivas (minería, petróleo y gas). Además, según un ranking general elaborado por Arellano Consultoría e Investigación de Mercados (2017), Alicorp es la mejor empresa para trabajar en el Perú del 2017 en el sector de consumo.

La importancia y relevancia de la marca fabricante Alicorp es innegable: es una marca de consumo masivo con un portafolio diverso dirigido a los segmentos $\mathrm{B}, \mathrm{C}$ y D principalmente, los segmentos más grandes en la población peruana, y con alcance a nivel nacional. Sus marcas compiten y lideran las categorías en las que se encuentran, haciéndole frente a grandes multinacionales como Procter \& Gamble, Kimberly Clark, Unilever, Colgate-Palmolive, Nestlé, entre otras. Algunas de estas marcas transnacionales trabajan (desde hace ya algunos años) para mejorar el propósito y la comunicación de sus marcas comerciales, apropiándose de luchas sociales como base para elaborar discursos publicitarios.

Por ejemplo, Procter \& Gamble, a través de sus marcas, establece claramente su rol de actor social: la marca Always, mediante la campaña "Like a girl" (2014) se ha apropiado del atributo del empoderamiento femenino-juvenil. Con la campaña "The best man can be" (2019), Gillette generó más de 35 millones de reproducciones, 800 mil "Me gusta” y más de un millón seiscientos mil "No me gusta" con su campaña basada en las masculinidades tóxicas (ver Figura 1). Con la campaña "The talk" (2017), H\&S trabaja a fondo el discurso para la comunidad afrodescendiente, utilizando hechos y comportamientos 
sociales como el tema del cabello afro y la rigidez con la que la policía suele tratar a los miembros de esta comunidad.

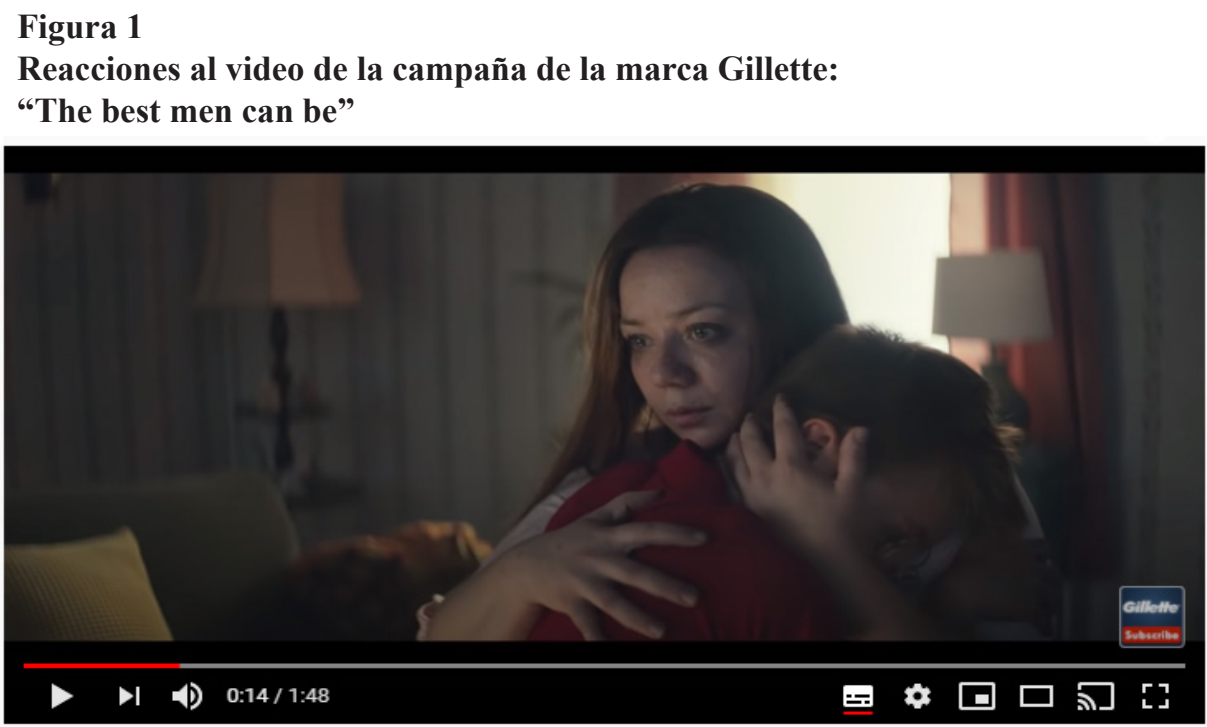

\#TheBestMenCanBe \#Gillette

We Believe: The Best Men Can Be I Gillette (Short Film)

35.203.880 visualizaciones $\cdot 13$ ene. 2019 if 818.004 q $1,6 \mathrm{M} \Rightarrow$ COMPARTIR $\equiv+$ GUARDAR $\ldots$

Nota: Gillette (2019).

La historia de la marca "Negrita" se remonta al año 1950 cuando la empresa ASA Alimentos lanzó esta marca comercial para los productos relacionados a la cocina y repostería: mazamorra, vainilla, chuño, entre otros, y fue comprada por Alicorp en el año 2006. Pero la marca "Negrita" tiene una fuerte asociación al producto mazamorra morada hecha de forma instantánea. La mazamorra era preparada por las mujeres negras esclavas para sus patrones y, según Ricardo Palma en sus Tradiciones Peruanas, durante la República la mazamorrera pregonaba a las siete de la noche, junto con el caramelero y la champucera, volviéndose así un postre popular y característico del país. Es de esta manera como este dulce se convierte en patrimonio cultural inmaterial del Perú.

El empaque de la marca muestra la imagen de una joven mujer negra con un pañuelo en la cabeza, un signo característico en las mujeres esclavas, quienes preparaban este postre para sus amos. A esta imagen le acompaña la palabra 
"Negrita" que hace referencia a una mujer de raza negra. Sin embargo, es la caracterización del personaje (en su representación gráfica, acompañada del nombre de la marca) la que mantiene el estereotipo de las mujeres afrodescendientes. Según Muñoz (2014) en la actualidad se reproducen los mismos roles del sistema esclavista que condiciona a las mujeres afrodescendientes a las actividades de cuidado: cocineras, lavanderas, etc. encasillándolas y limitando su acceso al mercado laboral calificado incluso hoy en día. (Muñoz, 2014).

Habiendo identificado la importancia de la marca fabricante y la descripción de la marca comercial, como antecedentes del caso, podemos comenzar a analizar el pronunciamiento de la empresa. El 22 de junio del 2020, Alicorp decide realizar un cambio de nombre sobre su marca comercial "Negrita", convirtiéndose en la primera empresa peruana que declara y tiene una posición frente al tema del racismo: su postura es clara sobre el tipo de actor social que quiere ser para la comunidad a la que ofrece sus productos. Este ha sido, sin duda alguna, un paso importante para las marcas nacionales dentro del contexto histórico y cultural del Perú.

Es así que, en medio de la campaña social \#blacklivesmatter, la empresa peruana Alicorp anunció en sus diversas redes sociales y en su página web oficial, el cambio de nombre e imagen de su marca "Negrita" para el primer trimestre del año 2021. En nuestro país, Alicorp es la primera y la única que se ha pronunciado y actuado para sentar un precedente de cambio y entendimiento de las demandas sociales de la comunidad afroperuana, ocasionando así diversos tipos de reacciones. Sin embargo, en nuestro análisis sobre dicho comunicado, vemos justificada esta decisión de cambio basándose en tres aspectos.

Primero, la empresa tiene una oportuna lectura del contexto. Esta se ve reflejada cuando mencionan que

la forma en que las sociedades abordan la diversidad y el racismo ha evolucionado en todo este tiempo ... aquello que antes podía considerarse positivo, hoy resulta inapropiado, pues todos somos más conscientes de que se consolidan estereotipos que buscamos superar... hemos visto lo que pasa en estos momentos en el mundo, en nuestra región y en el Perú. (Alicorp, 2020b) 
Segundo, la empresa asume su papel como actor social cuando afirma que

en el marco de nuestro compromiso con la diversidad y nuestra firme oposición al racismo en todas sus formas, hemos decidido que ha llegado el momento de cambiar ... el objetivo de esta iniciativa será encontrar una nueva forma de reconocer a todas las culturas y comunidades ... con un nombre y una identidad coherentes con nuestro respeto y valoración de la diversidad. (Alicorp, 2020b)

Tercero, la empresa muestra coherencia (entre el decir y el hacer) cuando proponen

jornadas de escucha con representantes de diversas comunidades para entender mejor su historia y preocupaciones actuales ... convocaremos también a especialistas externos para que nos ayuden a recorrer este camino en las diferentes decisiones que tomamos como compañía. (Alicorp, 2020b)

Esto evidencia que su propósito de marca tiene que estar relacionado con lo que quieren comunicar desde su nombre hasta la pieza más simple de comunicación, finalizando su comunicado de la siguiente manera: "Como parte de nuestro propósito de alimentar un mañana mejor, nos corresponde evolucionar para continuar inspirando respeto, inclusión y equidad, y así construir todos juntos la sociedad que queremos" (Alicorp, 2020b).

"Negrita" es una marca paraguas que le da nombre a varios productos, siendo el producto más recordado la mazamorra morada, un postre casero tradicional del Perú. Al ser "Negrita" una versión instantánea, podría considerarse un producto no esencial que no ha presentado desarrollo o mejoras. No obstante, es una marca emblemática y posee uno de los comerciales de televisión más recordados de los años noventa: "El chiquito mazamorrero". En una época donde la compra de medios estaba prácticamente monopolizada por la televisión y el zapping no existía, esta campaña estaba dirigida a jóvenes, niños y niñas que hoy son padres y madres de familia, público objetivo de la marca, parte de las generaciones X y millennial, quienes son los decisores de la compra y tienen actitudes favorables hacia las marcas con acciones coherentes y significativas. 
A pesar de que esta generación no esté estrechamente relacionada con las prácticas culinarias tradicionales del Perú, Negrita ha generado un vínculo emocional con sus clientes, apelando siempre al recuerdo de la infancia. Entonces, ¿por qué tomar esta decisión? Con el comunicado oficial de Alicorp, la marca fabricante logra poner sobre la mesa al racismo e induce a sus clientes a la reflexión de un futuro mejor, uno en el que se promueve la igualdad y no se perpetúa un estereotipo apoyándose meramente en la tradición. Por lo que hemos analizado, este cambio responde y es positivo para la marca Alicorp, pues la muestra coherente, con un propósito definido y siendo un actor con una posición clara sobre la discriminación racial. Además, le permite conectarse con sus públicos objetivos actuales: la generación $\mathrm{X}$ (que pasó por muchas carencias por la crisis económica de los ochentas y noventas, así como por el terrorismo) y la generación millennial, quienes (como hemos revisado en múltiples investigaciones) prefieren marcas con propósitos que aporten al desarrollo de la comunidad.

Es importante recalcar que el escenario del cambio de nombre podría haber sido otro. Alicorp pudo haber decidido no anunciar el cambio y efectuarlo directamente cuando lo creyera conveniente y, de esa manera, evitar la confrontación; sin embargo, optó por la comunicación abierta, donde compartió las diversas acciones alrededor del cambio de marca: charlas con gremios afrodescendientes y especialistas en el tema; un concurso público para buscar el nuevo nombre que represente a la marca, generándose así el diálogo, cumpliendo un rol y aprovechando la oportunidad. Algo que le da más peso a esta comunicación es la solidez empresarial y la visibilización que tiene la marca: Alicorp no es un actor social desconocido o insignificante. Por el contrario, se ha dado cuenta del poder que tiene sobre la población desde su calidad de anunciante con alcance a nivel nacional.

Ahora bien, el impacto positivo no es sólo sobre sus consumidores o sobre la posibilidad de poner a su marca como eje de la conversación mediática, sino que acciones como estas contribuyen al valor de marca (brand equity) y al fortalecimiento de la misma. Para Kotler \& Keller (2012), “el brand equity es el valor añadido que se asigna a un producto o servicio a partir de la marca que ostenta. Puede reflejarse en la forma en que los consumidores piensan, sienten y actúan con respecto a ella, así como en los precios, la participación de mercado y la rentabilidad que genera la marca para la empresa". Existen diversas formas de medir el brand equity y han ido variando con el tiempo; 
hoy se incluyen variables como relevancia, estima, rol de la marca y no sólo calidad, satisfacción y análisis financieros.

Según la metodología descrita en la web de la consultora internacional Interbrand (2020), esta se divide en tres grandes aspectos: a) financiero, medido en rentabilidad; $b$ ) el rol de la marca, como influencia de la marca sobre las decisiones de compra de los consumidores y c) la fortaleza de la marca, determinada por el grado de lealtad que puede desarrollar. Este último punto es medido mediante diez variables, de las cuales resaltaremos cuatro de estas, dos internas y dos externas:

Variables internas:

- Claridad: en lo que la marca representa con respecto a sus valores, posicionamiento y propuesta.

- Sensibilidad: como la capacidad de la marca para adaptarse y adelantarse a los cambios y oportunidades del mercado.

Variables externas:

- Autenticidad: basada en una verdad sólida y capacidades internas. Se evidencia al tener una historia definida y un conjunto de valores bien fundamentado.

- Presencia: determinada por la participación de la marca en el aquí y ahora a través de las referencias positivas entre sus consumidores, los líderes de opinión y la población.

Al tomar decisiones estratégicas en donde muestra claridad, autenticidad y coherencia sobre sus valores (así como sensibilidad y capacidad para reconocer, aceptar y adaptarse a los cambios sociales y a las nuevas demandas de los consumidores), Alicorp logra tener presencia en los medios de forma positiva y genera impacto en los consumidores y en los líderes de opinión. Todo este esfuerzo y trabajo se ven reflejados en el fortalecimiento de su marca. 


\section{Reacciones al cambio}

La postura adoptada por la marca en esta campaña resulta evidentemente retadora frente a la tradicional sociedad peruana. Por la reacción del público, en general, se puede evidenciar el rechazo a este cambio, que ha sido tomado como brusco y ofensivo por una buena parte de los ciudadanos. No obstante, ¿cuál es la postura que mantiene el gremio publicitario hacia este cambio y lo que esto significa? Según Tirado ${ }^{1}$ (2020), "si Alicorp tiene un propósito tan interesante y poderoso como 'Alimentar un futuro mejor', ¿por qué no extender esta actitud por cambiar los estereotipos en sus comunicaciones, en donde el espectador está más expuesto? Si esto no ocurre, el cambio de nombre de 'Negrita' podría quedar solo en la epidermis o en el gesto".

La reacción interpela a todo el gremio y lleva a una reflexión que propone un cambio mucho más grande y coherente, haciendo hincapié en la responsabilidad de los publicistas, en su calidad de comunicadores, de generar cambio en todos los ámbitos que les competan para que esta iniciativa no quede como un recuerdo, sino que sea el comienzo del cambio.

Flavio Pantigoso ${ }^{2}$ comentó que "a los afroperuanos se les ha encasillado en la publicidad como zapateadores, como mazamorreros o como gente que estaba en un rol de servidumbre. Ha sido una comunidad que ha sido relegada a roles muy estereotipados" (citado en Barrenzuela, 2020). Pantigoso vuelve a poner sobre la mesa el debate sobre la importancia del mensaje de esta campaña y, de manera muy similar a Tirado (2020), resalta la responsabilidad de Alicorp como agente activo de cambio social: "es una extraordinaria oportunidad para que Alicorp no solo cambie de nombre a su marca, sino que hable del problema del racismo, sobre la condición de los afroperuanos en el Perú ... y sería extraordinario ver a más exponentes de esa comunidad representados en la comunicación de la empresa" (citado en Barrenzuela, 2020).

Si bien son compartidas por muchas personas del gremio, sería poco realista pensar que las posturas de Tirado y Pantigoso representan la opinión del gre-

1. Director de la Asociación Peruana de Agencias de Publicidad.

2. Director de la agencia Zavalita Brand Building, ganador de 16 leones de Cannes y referente de la publicidad en el Perú. 
mio en total. Franco Tosso ${ }^{3}$ sostiene que "no hubo una mala fe en el nombre" y que si bien no considera que es agresivo tener la imagen y nombre que identifican a la marca en la actualidad, sí perpetúa estereotipos y "como comunicadores masivos estamos en capacidad de cambiar si queremos alcanzar la igualdad" (citado en Barrenzuela, 2020). Juan Carlos Gómez de la Torre ${ }^{4}$, publicista que fue parte del equipo que creó la campaña "Negrita sabe lo que te gusta" cuando esta marca aún le pertenecía a ASA Alimentos, explica que

"Negrita sabe lo que te gusta", fue la campaña que creamos por años con ASA Alimentos y convirtió a "Negrita" en líder de su categoría. "La Negrita" era muy inteligente, rápida, se adelantaba a los deseos de la gente. Por ejemplo, le quitó el logo de todos los artículos promocionales como vajilla, vasos, jarras. "La Negrita" le ganó a Yaps de Alicorp, Zuko de Tresmontes Chile, Tang de Kraft USA. "La Negrita" tenía una alta autoestima, se las sabía todas y nunca, nunca, se hubiera cambiado de nombre. (citado en Barrenzuela, 2020).

Por otro lado, tenemos a otro stakeholder importante: la prensa. Esta ha sido más bien tibia en su reacción hacia este cambio. Los tres principales medios del Perú (los diarios El Comercio, Trome y La República, los cuales reúnen casi el 55\% de alcance de lectoría) se limitaron a reproducir comunicados de prensa y realizar entrevistas a los expertos en el tema. De igual forma, las revistas y suplementos especializados desarrollaron notas desde el punto de vista del negocio, pero no determinaron una posición a favor o en contra. Si bien algunos medios le han otorgado a la noticia un matiz de nostalgia, no ha habido un juicio de valor concreto con respecto a las mismas.

La respuesta de la sociedad en los medios sociales no se hizo esperar, el anuncio de la marca en su cuenta oficial de Facebook (ver Figura 2) reúne más de mil quinientas reacciones, donde más de mil cien reacciones fueron de las expresiones "Me divierte" y "Me molesta" (reacciones con una connotación negativa) versus un poco más de cuatrocientas reacciones entre "Me gusta" y "Me encanta" (reacciones con una connotación positiva). La publicación

3. Director General Creativo en Fahrenheit DDB.

4. Publicista que cuenta con una amplia trayectoria que va desde CEO de la agencia Carne, hasta Director Creativo Ejecutivo en J. W. Thompson Worldwide. 
fue compartida más de 400 veces y se realizaron más de 900 comentarios, los que reflejaron una reacción de rechazo frente al cambio. En los comentarios publicados en dicha red, se justificó este rechazo usando argumentos como el de la "tradición", se negó la connotación racista $y$, en algunos casos, el pronunciamiento fue tildado de "oportunista".

\section{Figura 2}

Anuncio del cambio del nombre de "Negrita" en la cuenta oficial de Facebook

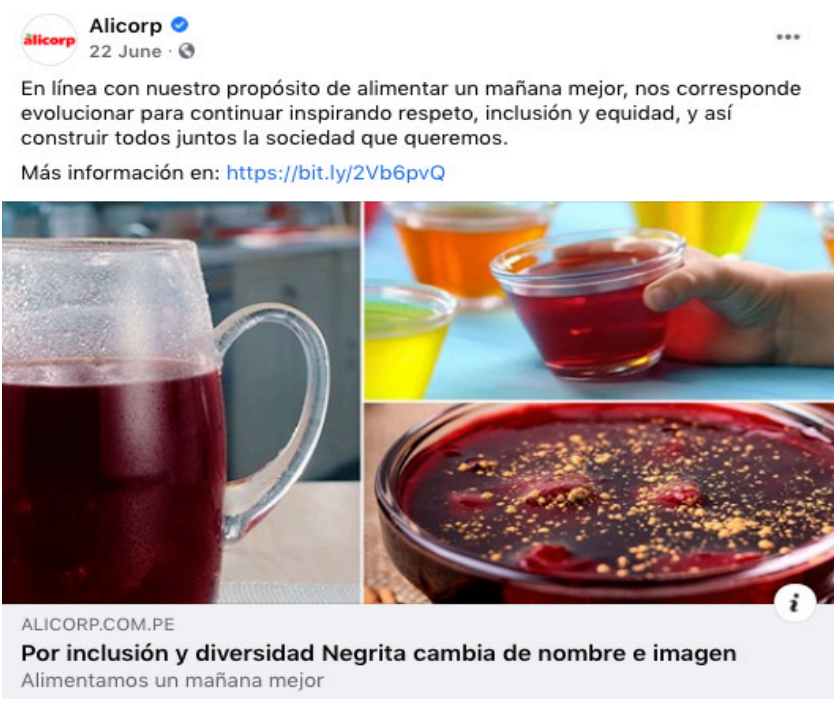

Nota: Alicorp (2020c).

Por otro lado, en LinkedIn (ver Figura 3) los números fueron distintos: la publicación se realizó desde la cuenta oficial de la empresa Alicorp, pero también fue publicada por el Chief Executive Officer de Alicorp, Alfredo Perez. En ambas cuentas se recibieron alrededor de 800 reacciones de las cuales más de 700 fueron positivas, entre "recomendar" y "celebrar". De igual forma, los más de 140 comentarios (en cada publicación) abordaban el tema y la reflexión desde diferentes aristas, siendo los comentarios opositores mayoritarios. 


\section{Figura 3}

Post de Alfredo Perez, CEO de Alicorp

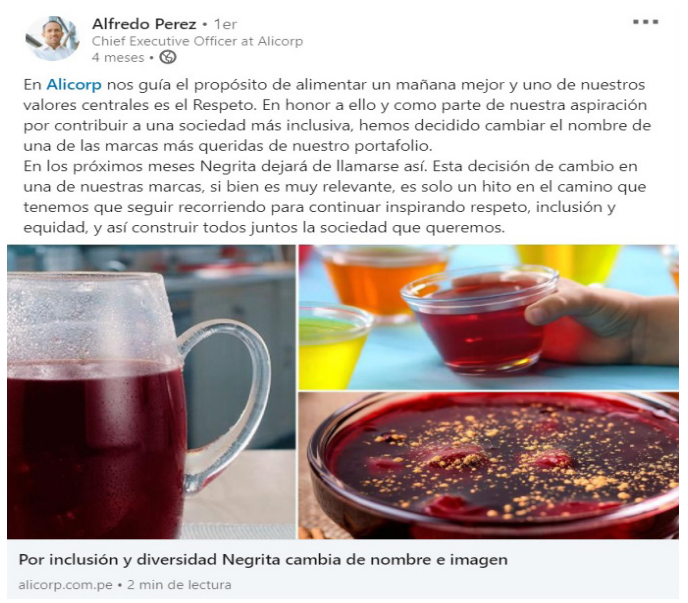

Nota: Pérez (2020).

Finalmente, en la cuenta oficial de la empresa en Twitter (ver Figura 4), la publicación contó con alrededor de 650 comentarios, más de 300 retweets y más de 800 "Me gusta". Los comentarios iniciaron el debate del racismo, la normalización de los estereotipos y cómo se utiliza la herencia cultural para darle legitimidad al statu quo instaurado sobre las representaciones de las personas afrodescendientes.

\section{Figura 4}

Reacciones del cambio del nombre de "Negrita" en la cuenta oficial de Twitter

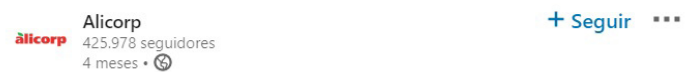

En línea con nuestro propósito de alimentar un mañana mejor, nos corresponde evolucionar para continuar inspirando respeto, inclusión y equidad, y así construir todos juntos la sociedad que queremos.

Más información en: https://bit.ly/2Vb6pvQ

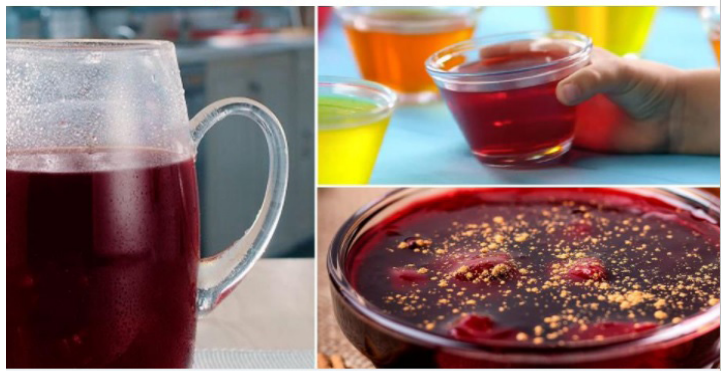

Por inclusión y diversidad Negrita cambia de nombre e imagen alicorp.com.pe $\cdot 2$ min de lectura

Nota: Alicorp (2020d). 
Por otro lado, los colectivos afrodescendientes (así como las organizaciones que defienden los derechos y la igualdad entre ciudadanos, como la Defensoría del Pueblo y el Ministerio de Cultura) se han pronunciado a favor de la marca. Incluso el Centro de Desarrollo Étnico (CEDET), invita a las marcas a sumarse a la iniciativa de Alicorp, es decir, a desarrollar publicidad que respete y ponga en valor los derechos humanos. Entre los líderes de opinión de la comunidad afroperuana destaca la periodista y activista de derechos humanos, Sofía Carrillo Zegarra. En una entrevista realizada en TVPerú (2020), Carrillo señaló que lo acontecido con la marca "Negrita" en el Perú, "corresponde a toda la propuesta que se tiene a nivel mundial con respecto a los estereotipos racistas que han estado cargando la publicidad ... Esto es sumamente importante" y resaltó que Alicorp está dando un mensaje positivo a los publicistas.

Los cambios suponen un proceso de aceptación y adaptación de los públicos involucrados. Interpelar la normalización de estereotipos raciales presenta desafíos, pero podemos ver que parte del rol que está asumiendo la marca es el de generador de conversación con respecto a un tema relevante en la coyuntura internacional que tiene, a su vez, un impacto en la situación actual de la comunidad afroperuana.

\section{Discusión: demandas a la publicidad}

La politización de las marcas es un fenómeno del que los consumidores estamos siendo gestores. Las exigencias de los consumidores han cambiado. Ya no sólo buscan un producto de buena calidad, sino que ahora buscan también que este se posicione como un agente de cambio y muchas veces exige que este cambio sea político. Bajo el concepto de que el sector privado se encuentra en una posición de ventaja sobre el público, los consumidores demandan que este participe también dentro del debate del cambio social ya que sus productos e ideas impactan los hábitos, costumbres y prejuicios de la sociedad.

En definitiva, las medidas que puede tomar una marca comercial (con respecto a temas que abordan problemas sociales) suelen ser incómodos para las personas y entidades. Es una decisión impopular que puede afectar la imagen de la marca. Sin embargo, también puede ser una oportunidad para liderar la conversación entre los diversos stakeholders de la misma. 
Volviendo al caso de "Negrita", podemos ver cómo una marca bien posicionada en el mercado (a pesar de la poca inversión publicitaria durante varios años) puede lograr alcance, interacción y volverse relevante. Esta se presenta como la posibilidad de traer al primer plano de la escena comercial una marca posicionada (pero sin inversión publicitaria durante varios años) y así lograr alcance, interacción y volverse relevante. "Negrita" mantiene en alerta a su audiencia hasta el día de la transformación total con la adopción del nuevo nombre y nueva identidad gráfica prometida para el primer trimestre del año 2021. Mientras tanto, se apodera de una causa que alimenta la coherencia en relación a su propósito como marca fabricante y crea la conexión directa entre "el decir y el hacer" que tanto reclaman y observan las personas, cuando se trata de juzgar una empresa-marca. Debido a esto, es importante prestar atención a las demandas actuales que personas-consumidores exigen y esperan de las empresas-marcas:

\subsection{Inclusión como representatividad}

Al hablar de la representatividad de los grupos minoritarios en la publicidad, se puede decir que

el marketing inclusivo se esfuerza por crear una cultura visual más representativa. Se esfuerza por apreciar y comprender diversas identidades, diferencias e historias, al mismo tiempo que enfatiza los lugares comunes. Los especialistas en marketing inclusivo están dispuestos a cultivar el conjunto de habilidades necesarias para promocionar sus productos según datos demográficos específicos sin depender de estereotipos. El marketing inclusivo adopta una posición progresista en cuestiones de justicia. (Fish, 2016)

En este sentido, dentro del ámbito publicitario local, ha comenzado a gestarse un camino hacia la promoción de productos desligada de estereotipos y propenso a generar un cambio estructural de lo se presenta como publicidad tradicional. Atrás quedan los antiguos paradigmas dictados por la norma general, la cual propone ideas sin asumir responsabilidades de las repercusiones en la sociedad. 


\subsection{Igualdad en los roles}

A pesar de los diferentes esfuerzos actuales por generar un cambio global sobre estereotipos de género, estos siguen siendo usados en el campo de la publicidad y muchas marcas se niegan a aceptar el cambio por miedo a perder la fidelidad de sus consumidores hacia las marcas:

Los críticos afirman que la publicidad no refleja el avance significativo del movimiento por la igualdad de género en muchas sociedades. Básicamente, hay dos conclusiones generales: pesimista y optimista. Los estudios pesimistas enfatizan que las mujeres todavía están siendo retratadas de manera negativa y que, por ende, este tipo de estereotipos está incluso empeorando. Por otro lado, los estudios optimistas consideran que las mujeres se encuentran cada vez más cerca de sus homólogos masculinos y que, por ende, se van rompiendo los estereotipos negativos. Además, proponen que la publicidad está comenzando a mostrar la imagen de la mujer contemporánea y que poco a poco van tomando la misma importancia que los hombres. (Eisend, 2009)

Se habla mucho del "efecto espejo" (Holbrook \& Westwood, 1989) afirmándose que la publicidad refleja los valores existentes. Podemos decir que en este contexto de cambio, el reflejo de las expectativas de la sociedad podría ser algo positivo y que ayude a que este se geste de una manera más rápida.

\subsection{Estereotipos positivos}

Por muchos años, la publicidad ha sido criticada por perennizar diversos estereotipos de género, raza y condición social. Ejemplos sobran en el rubro "cuidado de personas" y “cuidado del hogar". El caso peruano no es la excepción: campañas vinculadas al tema de la cocina presentaban a la típica ama de casa con el esposo proveedor, el sabor de la comida (y el baile) asociados a la comunidad afroperuana y los roles de la asistencia en el hogar para las mujeres andinas.

Vale la pena precisar que, bajo la definición de diversos autores, la publicidad sí necesita trabajar con estereotipos. Con esto nos referimos a la definición de Eirini \& Zotos (2014), quienes proponen que 
las creencias de que ciertos atributos diferencian a mujeres y hombres...existen cuatro componentes diferentes e independientes: descriptores de rasgos (por ejemplo, autoafirmación, preocupación por los demás), características físicas (por ejemplo, largo del cabello, altura del cuerpo), comportamientos de rol (por ejemplo, líder, cuidado de niños) y profesión (por ejemplo, conductor de camión, ama de casa). Cada componente está asociado con una versión masculina y femenina, que está fuertemente relacionada con hombres y mujeres.

Por ello, cuando se define el público objetivo (al cual estará dirigido un producto o servicio) se segmenta a la población y se elige las diversas características sobre las cuales se va a construir la representación de estos en las campañas publicitarias y sus diversos medios de difusión. Sin embargo, el escenario actual es otro: así como la situación de las campañas, las personas demandan que los estereotipos negativos, limitados y discriminadores sean reemplazados por aquellos que muestran otros aspectos de las personas. Estas quieren ver todos los roles que pueden representar en la sociedad reflejados en los medios. Una mujer no es solo madre, también es empresaria, estudiante, es amiga, es deportista, etc., y estos roles son el resultado de un esfuerzo personal que va de la mano con los cambios sociales.

Además, sabemos que (desde el análisis del comportamiento del consumidor) cada individuo cumple diversos roles en sus interacciones comerciales, dependiendo del producto o servicio al que se haga referencia. Podemos ser consumidores, decisores o influenciadores y, a la vez, estos roles se cruzan con los roles familiares o sociales que adquirimos en la evolución de nuestro ciclo de vida. En síntesis, los individuos son mucho más que un solo estereotipo.

\section{Conclusiones}

Podemos ver que la publicidad ha normalizado muchas representaciones de estereotipos que contribuyen a la asignación de roles y espacios a los grupos minoritarios: mujeres, afrodescendientes, homosexuales, entre otros. Sin embargo, algunas acciones realizadas por las marcas (así como reflexiones generales del gremio publicitario) invitan a repensar la comunicación publicitaria como parte intrínseca del discurso político de una marca. 
El escenario actual, el contexto, el fortalecimiento de la sociedad civil organizada y el equilibrio de poder (a través del alcance con la comunicación digital) enfrentan a las marcas con la urgente tarea de dejar de moverse en el margen del conflicto social y tomar una participación activa y retadora.

La transformación oportuna de los discursos de marca resulta una tarea imperativa. Una marca no puede canalizar todos sus esfuerzos en crear un vínculo sólo con sus consumidores: esto ya no es suficiente. Ahora se le exige ir mucho más allá. Debe identificar, conectar y conversar con todos sus públicos, desde sus consumidores más fieles hasta los stakeholders que se creían lejanos y sin ningún impacto sobre esta.

Asimismo, las empresas deben asumir su rol como actores sociales trascendentes y entender que los resultados de sus acciones son directamente proporcionales a estas. Cuanto mayor sea el alcance geográfico poblacional de sus productos o servicios y cuanto mayor sea su inversión en comunicación publicitaria, será mayor su responsabilidad para contribuir en la difusión de representaciones positivas de las minorías y mayor será su influencia sobre la sociedad.

No todas las marcas tienen la oportunidad ni las plataformas para generar un debate. No todas saben cómo tomar ventaja de un momento para alinearlo con su propuesta comercial, su propósito de marca o su público objetivo. Sin embargo, como hemos visto, las que logren manejarse adecuadamente (dentro de un terreno tan delicado) serán parte del primer grupo gestor del cambio y serán las primeras en lograr este nuevo posicionamiento de marca.

Dada la naturaleza dinámica de los cambios políticos y sociales, es importante que las marcas fabricantes mantengan un monitoreo continuo frente a la potencial influencia sobre sus productos. Así, el reto es lograr un equilibrio adecuado entre la participación activa en los cambios sociales constantes, los lineamientos de la marca y las nuevas exigencias de los consumidores. 


\section{Fuentes consultadas}

Aaker, J., Fournier, S., \& Brasel, A. (2004). When good brands do bad. Journal of Consumer Research, 31, 1- 25. https://doi.org/10.1086/383419

Aaker, D (2010). Building strong brands. Pocket.

Ahluwalia, R., Swaminathan, V., \& Stilley, K. (2009). When Brand Personality Matters: The Moderating Role of Attachment Styles. Journal of Consumer Research, 35, 985-1002. https://doi.org/10.1086/593948

Ahuvia, A. (2005). Beyond the Extended Self: Loved Objects and Consumers. Identity Narratives, Journal of Consumer Research, 32, 171184. https://doi.org/10.1086/429607

Alicorp. (2018). Reporte anual 2018. https://www.alicorp.com.pe/media/ PDF/Alicorp_Reporte_2018_compressed_1_W28nuxu.pdf

Alicorp. (2019). Memoria anual consolidada 2019. https://www.alicorp.com. pe/media/PDF/Memoria_Anual_Consolidada_2019.pdf

Alicorp. (2020a). Alicorp cierra el 2019 con resultados positivos. https:// www.alicorp.com.pe/pe/es/noticias/alicorp-cierrael-2019-con-resultados-positivos/

Alicorp. (2020b). Comunicado de cambio de nombre de marca Negrita. https://www.alicorp.com.pe/pe/es/noticias/porinclusion-y-diversidadnegrita-cambia-de-nombre-e-imagen/

Alicorp (2020c, Junio 22). Por inclusión y diversidad Negrita cambia de nombre e imagen [Post]. Facebook. https://www.facebook. com/359140338272499/posts/706774013509128/?sfnsn=mo

Alicorp (2020d, Junio 22). Por inclusión y diversidad Negrita cambia de nombre e imagen [Tweet]. Twitter. https://www.linkedin.com/posts/ alicorp-saa_por-inclusi\%C3\%B3n-y-diversidad-negrita-cambia-activity-6680854173208121344-QY1B 
América Economía. (2019). Estas son las 500 empresas más grandes del Perú 2019. https://www.americaeconomia.com/negocios-industrias/ estas-son-las-500empresas-mas-grandes-del-peru-2019

Antón, R. (2015). Participación horizontal y abierta en la red: Cocreación en racimos. http://e-spacio.uned.es/fez/eserv/tesisuned:Educacion-Ranton/ANTON_CUADRADO_Raul_Tesis.pdf

Aron, A., \& Aron, E. (1996). Love and the expansion of the self: The state of the model. Journal of Analytical Psychology, 3, 45-58. https://doi. org/10.1111/j.1475 6811.1996.tb0 0103.x

Arellano Consultoría e Investigación de Mercados. (2017). Aportes a los cambios sociales. https://www.arellano.pe/aportes-los-cambios sociales/

Arellano, R. (2017, Noviembre 13). Dónde quiero trabajar 2017. El Comercio. https:/elcomercio.pe/opinion/rincon-del-autor/quiero-2017-rolando-arellano-noticia-473329-noticia

Avery, J., Breazeale, M., \& Fournier, S. (2015). Strong brands, strong relationships. Routledge, Taylor \& Francis Group.

Barrenzuela, I. (2020). ¿Qué opinan los líderes de la industria sobre el rebranding de 'Negrita'? Mercado Negro. https://www.mercadonegro.pe/branding/que-opinan-los-lideres-de-la-industria-sobre-el-rebranding-de-negrita/

Bechara, A., Castaño, R., Reimann, M., \& Zaichkowsky, J. (2012). How we relate to brands: Psychological and neurophysiological insights into consumer-brand relationships. Journal of Consumer Psychology, 22, 128-142. https://doi.org/10.1016/j.jcps.2011.11.003

Blackston, M. (1992). Observations: building brand equity by managing the brand's relationships. Journal of Advertising Research, 32, 79-83. https://doi.org/10.2501/JAR-40-6-101-105

Breazeale, M., Fetscherin, M., \& Fournier, S. (2012). Consumer-brand relationships: Theory and practice. Routledge. 
Bruns, D., Fischer, A., Langner. T., \& Rossiter, J. (2014). Falling in love with brands: a dynamic analysis of the trajectories of brand love. Marketing Letters, 27, 15-26. https://doi.org/10.1007/s11002-014-9283-4

Castells, M. (1997). Local y global. La gestión de las ciudades en la era de la información. Taurus.

Centro de Desarrollo Étnico. (2020). Caso producto "Negrita". http://cedetperu.org/index.php/prensa/item/145-pronunciamiento-caso-producto-negrita

Dafonte-Gómez, A. (2014). Claves de la publicidad viral: De la motivación a la emoción en los videos más compartidos. Comunicar. Media Education Research Journal, 22, 199-206. https://doi.org/10.3916/C432014-20

Eirini, T., \& Zotos, Y. (2014). Female stereotypes in print advertising: A retrospective analysis. Procedia. Social and Behavioral Sciences, 148, 446-454. https://doi.org/10.1016/j.sbspro.2014.0 7.064

Eisend, M. (2009). A meta-analysis of gender roles in advertising. Journal of the Academy of Marketing Science, 38, 418-440. https://doi.org/10.10 80/00913367.2019.1566103

Esté, A. (2020, Julio 9). De Aunt Jemima a Juan Valdez: cómo reinventar las marcas en tiempos de crisis. New York Times. https://www.nytimes. com/es/2020/07/09/espanol/opinion/marcas-racismo-latino-america. html

EY Beacon Institute. (2017). How can purpose reveal a path through disruption? Mapping the journey from rhetoric to reality. https://www. ey.com/Publication/vwLUAssets/ey-how-can-purpose-reveal-apath-through-uncertainty/\$File/ey-how-can-purpose-reveal-a-paththrough-uncertainty.pdf

Felder, D. (1999). Comunicación para el cambio social. http://grupocomunicaciones.files.wordpress.com/2009/02/comunicacion_para_el_cambio_social.pdf 
Fish, J. (2016) Make an impact. Your guide to inclusive marketing. https:// www.forbes.com/sites/womensmedia/2016/06/29/make-an-impactyour-guide-toincl usive-marketing/

Fournier, S. (1994). A consumer-brand relationship framework for strategic brand management [Tesis doctoral]. University of Florida. https:// ufdc.ufl.edu/AA00038629/00001

Fournier, S. (1998). Consumers and their brands: Developing relationship theory in consumer research. Journal of Consumer Research, 16, 343353. https://doi.org/10.1086/209515

García Avilés, J. (2015). Comunicar en la sociedad red. Teorías, modelos y prácticas. UOC.

Gillette. (2019, Enero 13). We Believe: The Best Men Can Be | Gillette (Short Film) [Video]. YouTube. https://www.youtube.com/watch?v=koPmuEyP3a0

Holbrook, M., \& Westwood, R. (1989). The role of emotion in advertising revisited: Testing a typology of emotional responses. En P. Cafferata \& A. Tybout (Eds.), Cognitive and affective responses to advertising (pp. 353-371). Lexington Books.

Kelly, S. (2012). Examining the role of perceived immediacy as a mediator: Revisiting the relationships among immediate behaviors, liking, and disclosure [Tesis doctoral]. University of Tennessee. https://trace.tennessee.edu/utk_graddiss/1414/

Kotler, P., \& Keller, K. (2012). Marketing Management. Pretince Hall, Pearson.

Livingstone, S (2019). What brand purpose really means and why it matters. https://blog.globalwebindex.com/marketing/brandpurpose/

Llorca, G., Cano, L. (2016). Espacio y tiempo en el siglo XXI: velocidad, instantaneidad y su repercusión en la comunicación humana. Revista Cientifica de Comunicación, 6, 219-233. http://www.comhumanitas. org/index.php/comhumanitas/article/view/201516 
Interbrand. (2020). Home page. https://www.interbrand.com

IPSOS. (2018). 1 Encuesta nacional. Percepciones sobre diversidad cultural y discriminación ético racial. https:/www.ipsos.com/sites/default/files/ct/news/documents/201803/percepciones-sobre-diversidad-culturalydiscriminacion-etico-racial.pdf

IPSOS. (2019). Peruano pingüino. https://www.ipsos.com/es-pe/peruano-pingüino

Ministerio de Cultura del Perú. (2018). Resultados de la I Encuesta Nacional Percepciones y actitudes sobre diversidad cultural y discriminación étnico-racial. https://www.gob.pe/institucion/cultura/ noticias/4975-un-53-considera-que-peruanosson-racistas-o-muy-racistas-pero-solo-el-8-se-percibe-asimismo-comoracistaomuy-racista

Media Ownership Monitor Peru, MOM. (2018) Impresos. https://peru.momrsf.org/es/medios /prensa

Miranda, O. (2020). Las "negritas" de la publicidad peruana. La República. https://larepublica.pe/domingo/2020/06/28/las-negritas-de-la-publicidad-peruana/

Mobolade, O. (2016). How to market effectively to Millennials. Warc best practice. https://www.iabswitzerland.ch/wpcontent/uploads/2016/06/ millwardbrown_article_how-to-market-effectively-to-millenn ials.pdf

Monitor Empresarial de Reputación Corporativa. (2019). Ranking de evaluación reputacional 2019. https://www.merco.info/pe/ranking-merco-empresas

Muñoz, R. (2014). Afroperuanas. Situación y marco legal de protección de sus derechos. https://centroderecursos.cultura.pe/sites/default/files/rb/ pdf/05.pdf

Navarro, M. (2020). Comunicación horizontal e interactividad. En J. Figuereo (Ed.), Estudios multidisciplinarios en comunicación audiovisual, interactividad y marca en la red (pp. 97-112). Egregius. 
Pérez, A. (2020, Junio 22). Por inclusión y diversidad Negrita cambia de nombre e imagen [Tweet]. Twitter. https://www.linkedin.com/posts/ alfredo-perez-65312273_por-inclusi\%C3\%B3n-y-diversidad-negrita-cambia-activity-6680852523424735232-arUa

Perú Retail (2017). Interbank y Alicorp son reconocidas como las mejores empresas para trabajar en el Perú. https://www.peru-retail.com/interbank-alicorp-reconocidas-mejores-empresas-trabajar-peru/

Raffino, M. (2020) Comunidades virtuales. https://concepto.de/comunidadesvirtuales/\#ixzz6WO5UcIje

Rushkoff, D. (1994). Media virus. Ballantine.

Sainz de Medrano, V. (2017). De vertical a horizontal. De la comunicación de masas a la comunicación masiva [Tesis Doctoral]. Universidad Complutense de Madrid, Madrid. https:/eprints.ucm.es/41708/1/ T38559.pdf

Schwigel, C. (2019, Junio 13). Comunidades digitales y comunidades humanas: las redes y la importancia del rol de comunicadores y constructores sociales. http://signisalc.org/noticias/articulos/13-06-2019/ comunidades-digitales-y-comunidades-humanas-las-redes-y-la-importancia-del-rol-de-comunicadores-y-constructores-sociales

Segarra-Saavedra, J., \& Hidalgo-Marí, T. (2018). Viralidad e interacción. Análisis del engagement de los diez anuncios más vistos en YouTube en España en 2016. ICONO14. Revista Cientifica de Comunicación y Tecnologías Emergentes, 16(1), 47-71. https://doi.org/10.7195/ri14. v16i1.1069

Sibilia, P. (2006). El hombre postorgánico: cuerpo, subjetividad y tecnologías digitales. Fondo de Cultura Económica.

Tirado, J. (2020). Caso Negrita: La oportunidad más allá del cambio de nombre. https://www.mercadonegro.pe/columna/caso-negrita-la-oportunidad-mas-alla-del-cambio-de-nombre/ 
TVPerú Noticias [@perunoticias]. (2020, Junio 22).Marca Negrita cambiará de nombre [Imagen adjunta] [Tweet]. Twitter. https://twitter.com/notic ias_tvperu/status/1275412586025168899

Uche, S. (2018). Generation Z and Corporate Social Responsibility [Tesis de maestría]. Syracuse University. https://surface.syr.edu/thesis/226/ 